\title{
Effect of Curcumin and Low Frequency Electromagnetic Field on the Hormones of Pituitary- Gonad Axis in Male Diabetic Rats
}

\section{ARTICLE INFO}

\section{Article Type}

Original Research

\section{Authors}

Sadoughi S.D.* $P h D$

How to cite this article
Sadoughi S.D. Effect of Curcumin
and Low Frequency
Electromagnetic Field on the
Hormones of Pituitary-Gonad
Axis in Male Diabetic Rats.
Horizon of Medical Sciences.
2017;23(1):27-33.

*Young Researchers and Elite Club, Mashhad Branch, Islamic Azad University, Mashhad, Iran

\section{Correspondence}

Address: Biology Department, Sciences Faculty, Payam-e-Noor University, Mo'allem Boulevard, Mashhad, Iran. Postal Code: 91735-433 Phone: +98 (51) 38683900 Fax: +98 (51) 38683001 damoon.sadoughi@mshdiau.ac.ir

\section{Article History}

Received: January 26, 2016

Accepted: July 19, 2016

ePublished: January 19, 2017

\section{A B S T RACT}

Aims Changing the activity of pituitary-testis axis, hyperglycemia leads to fertility disorders. Curcumin reduces blood sugar, and the electromagnetic fields differently affect the cells and tissues. The aim of the study was to investigate the effects of curcumin and the low-frequency electromagnetic fields on the activities of pituitary-gonad axis in the diabetic male rats. Materials \& Methods In the experimental study, 49 Wistar male rats were studied. The rats were randomly divided into seven groups ( $n=7$ rats per group), including healthy control, diabetic control (receiving distilled water), experimental diabetic $1(300 \mathrm{mg} / \mathrm{Kg}$ curcumin as intraperitoneal injection), experimental diabetic 2 (curcumin +60 -minute $50 \mathrm{~Hz}$ and $15 \mathrm{G}$ electromagnetic field daily), experimental diabetic 3 (curcumin $+50 \mathrm{~Hz}$ and 300G electromagnetic field), experimental diabetic 4 (50Hz and $15 \mathrm{G}$ electromagnetic field), and experimental diabetic $5(50 \mathrm{~Hz}$ and $300 \mathrm{G}$ electromagnetic field) groups. One alloxan intraperitoneal injection was used to cause diabetes. 25-day treatment was conducted in all the groups. At the end of treatment period, the serum levels of testosterone, dihydrotestosterone, estrogen, LH, and FSH hormones were measured. Data was analyzed by SPSS 20 software using one-way ANOVA and Tukey post-hoc tests.

Findings The serum levels of all the studied hormones significantly increased in experimental diabetic groups 1,2 , and 4 compared to diabetic control group, while they significantly decreased in experimental diabetic group $5(\mathrm{p}<0.05)$.

Conclusion Curcumin increases the activity level of pituitary-testis axis in the diabetic male rats. The effects intensify by the simultaneous $15 \mathrm{G}$ electromagnetic field.

Keywords Diabetes; Curcumin; Electromagnetic Fields; Testis; Pituitary-Gonad Axis; Rats

\section{CITATION LINKS}

[1] Obesity and diabetes mellitus in ... [2] Investigating the effects of aqueous extract of ... [3] Effect of pentoxifylline on Serotoli and ... [4] Molecular regulation of ... [5] Hormonal regulation of ... [6] Correlation between spermatogenesis disorders and rat ... [7] Effect of Spirulina maxima on ... [8] Diabetes, oxidative stress and therapeutic ... [9] Testosterone, diabetes mellitus, and ... [10] Protective mechanism of ... [11] Prevention of diabetes by thymic hormone in ... [12] the effect of ... [13] Magnetic field exposure during gestation: pineal and cerebral ... [14] Effects of $900 \mathrm{MHz}$ electromagnetic field on ... [15] Investigating the effects of ... [16] Investigating the effects of low-frequency ... [17] Investigating the effects of ... [18] Investigating the effect of electromagnetic field with low ... [19] Effects of extremely low frequency ... [20] Effect of a 50-Hz electromagnetic field on the gene expression of glutathione s-transferase ... [21] Biological and Anticancer Effects of ... [22] Multiple biological activities of ... [23] Curcumin and cancer: An "oldage" disease with an ... [24] Antioxidant and anti-inflammatory potential of curcumin accelerated the cutaneous wound healing in ... [25] Antioxidative components isolated from rhizome of ... [26] Protective effect of curcumin on the ... [27] The effect of curcumin on ... [28] Effect of curcumin on angiogenesis in ... [29] The comparison of ... [30] "Spicing up" of the immune system by ... [31] The effect of curcumin on serum level of ... [32] Effect of aqueous extract ... [33] Influence of the hypothalamic-pituitary-adrenal axis ... [34] Possible neuroprotective mechanisms of ... [35] Evaluation of the nitric oxide ... [36] Antidiabetic effect of curcuma longa ... [37] Effect of curcumin supplementation on ... [38] Curcumin extract for ... [39] Evaluation of Turmeric (Curcuma longa) effects in ... [40] Effect of curcumin on ... [41] Ameliorative effects of curcumin on ... [42] The effect of low-frequency electromagnetic fields on ... [43] DNA fragmentation in human fibroblasts under ... [4.4] Effects of low frequency electromagnetic fields on ... [45] Curcumin induces glutathione ... [46] Introduction to ... [47] Effect of electromagnetic field on ovary and ... 
ترشح آندروزنها، بسيارى از جنبههاى فيزيولوزيك فرد از جمله تمايز

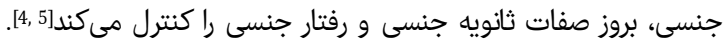

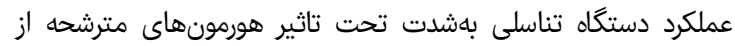

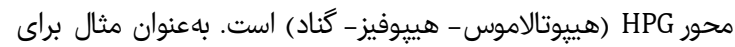

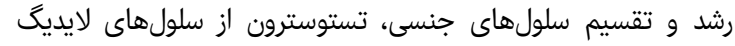

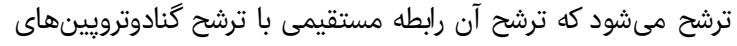

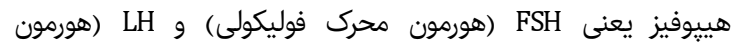
لوتئينىكننده) و هورمون GnRH (هورمون آزادكنيد گكنادوترويين) (هرون) هييوتالاموس دارد[5].

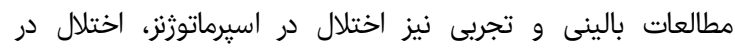

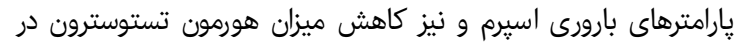

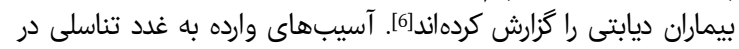

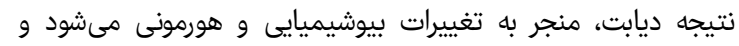

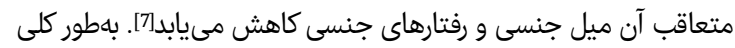

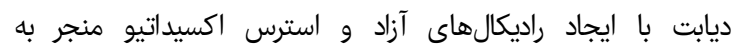

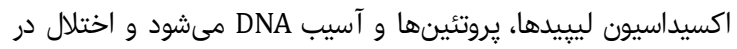

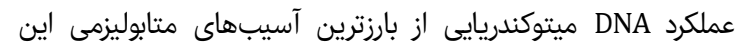

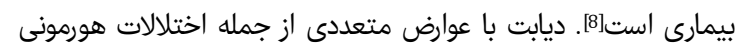

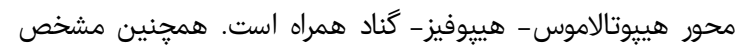

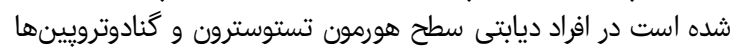

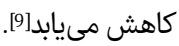

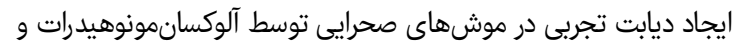

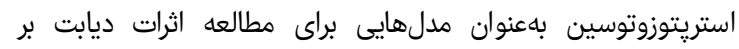

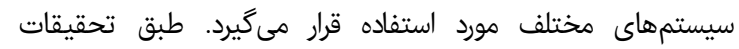

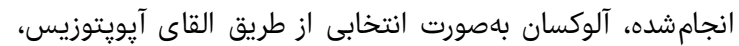

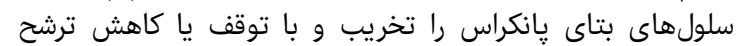

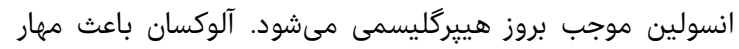

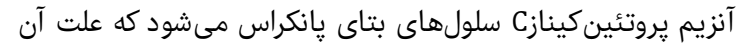

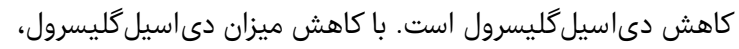

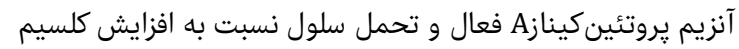

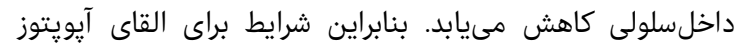

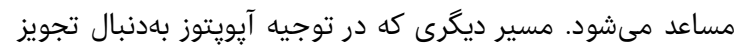

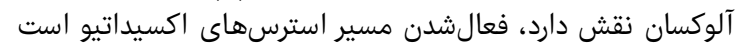

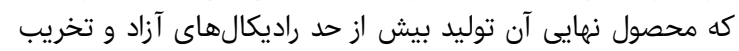

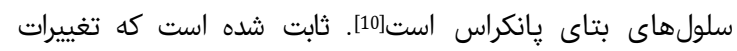

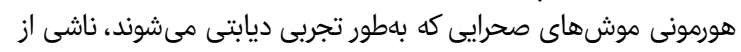

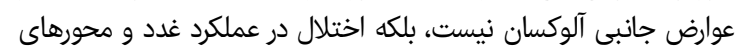

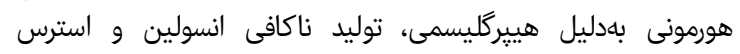

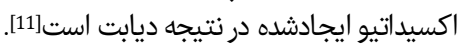

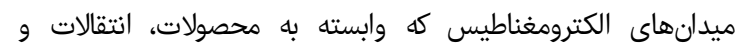

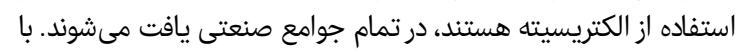

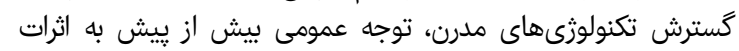

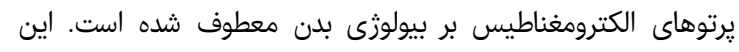

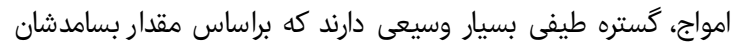

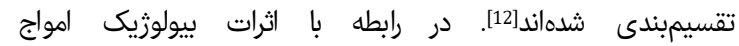

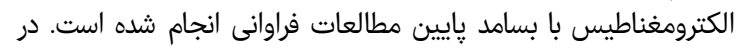

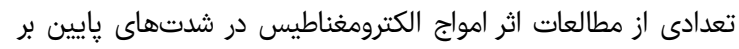

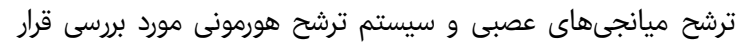

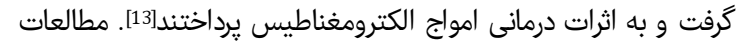

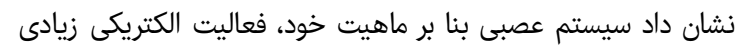

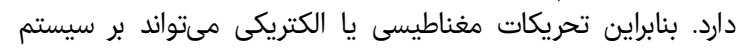
عصبى تاثيرگذار باشد.
اثر كوركومين و ميدان الكترومغناطيس با بسامد

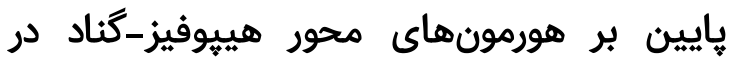
موشهاى صحرايى نر ديابتى
سيدامون صدوقى" PhD باشكًاه يزوهشكًان جوان و نخبكان، واحد مشهد، دانشكاه آزاد اسلامى، مشهد، ايران

جكيده اهداف: هيبركليسمى با تغيير فعاليت محور هييوفيز- بيضه منجر به اختئل اختلال در

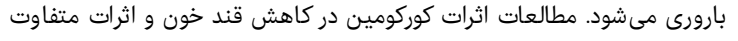

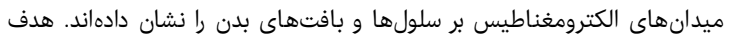

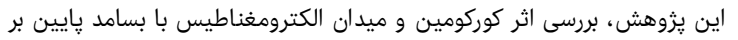

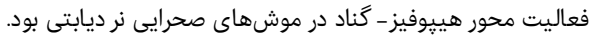

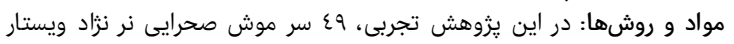

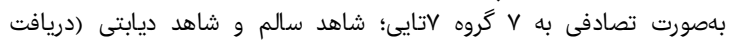

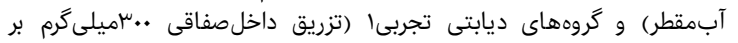

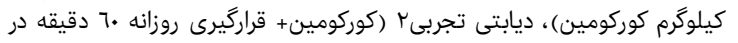

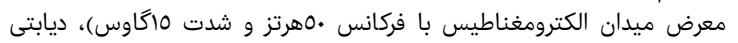

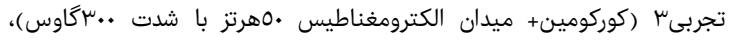

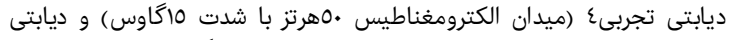

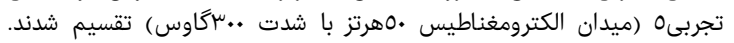

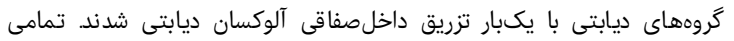

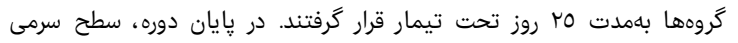

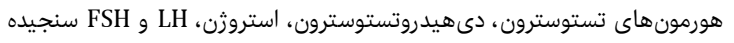

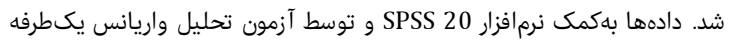

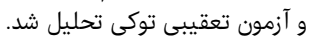

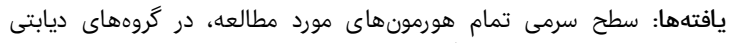

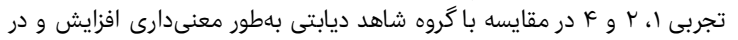

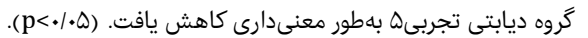

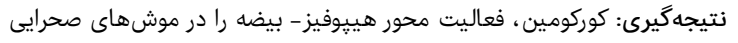

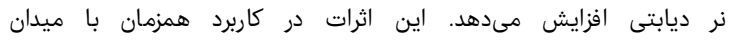

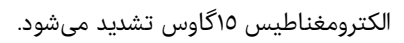

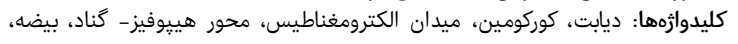

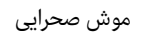

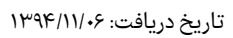

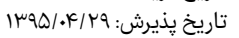

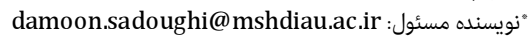

\section{مقدمه - مقد}

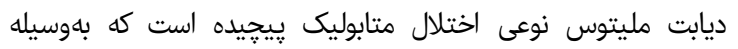

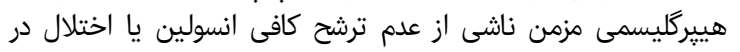

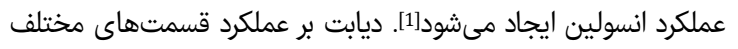

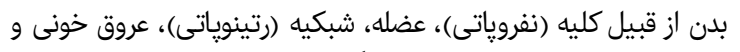

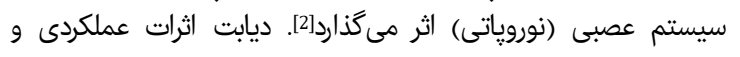

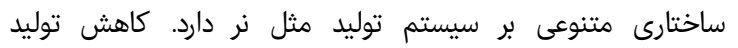

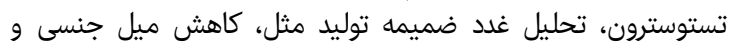

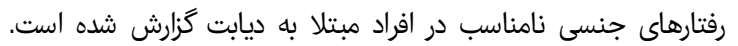

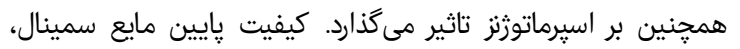

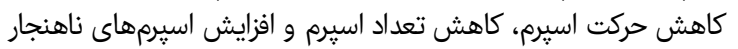

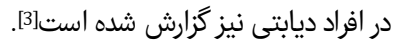

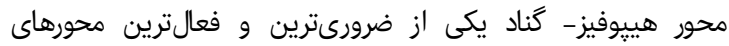
فيزيولوزيك بدن است كه نهتنها اعمال توليد مثلى، بلكه بهواسطه سنتز و 


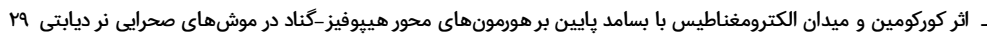

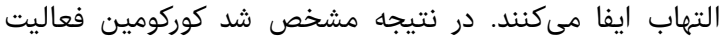

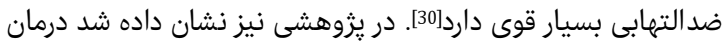

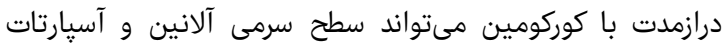

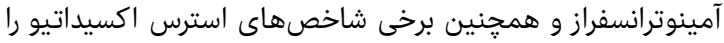

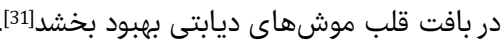

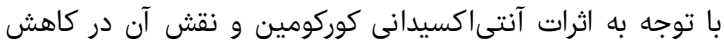

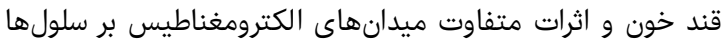

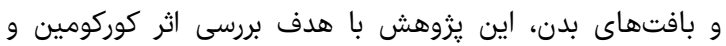

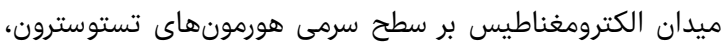

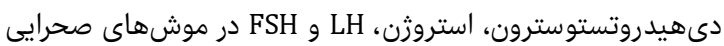

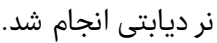

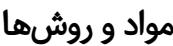

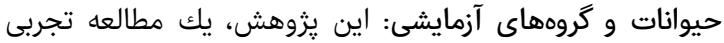

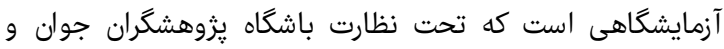

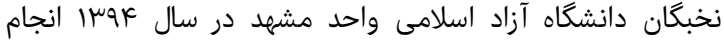

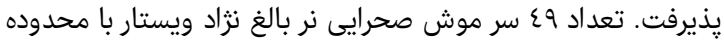

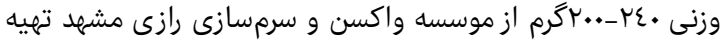

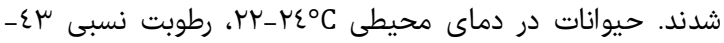

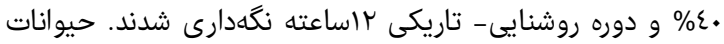

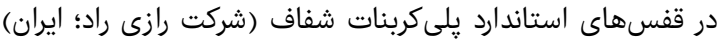

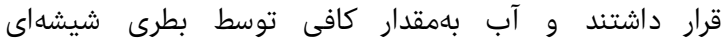

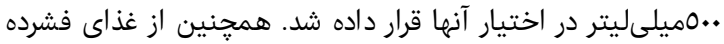

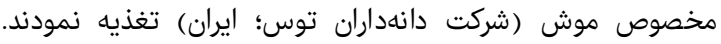
بهمنظور حصول حالت سازش با محيط، تمامى آزمايشها

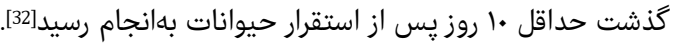

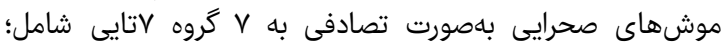

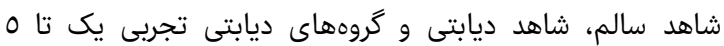

تقسيم شدند.

نحوه تيمار گروهها باهصورت زير بون بود:

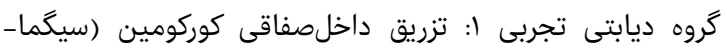

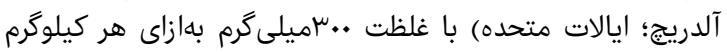

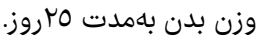

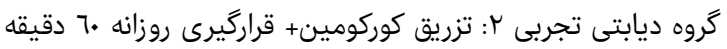

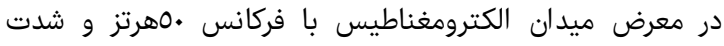

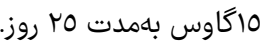

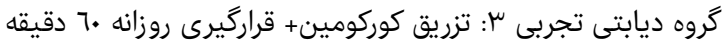

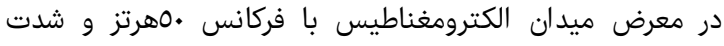

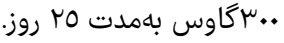

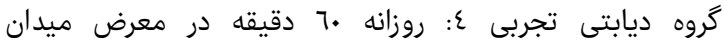

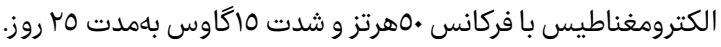

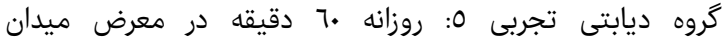

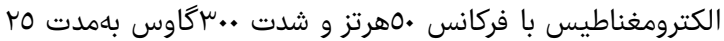

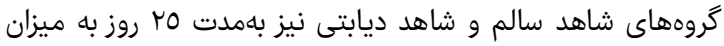

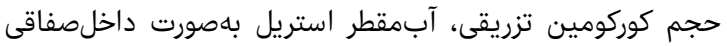

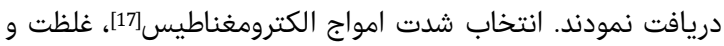

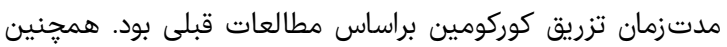

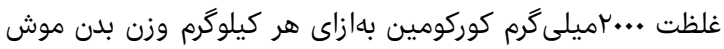

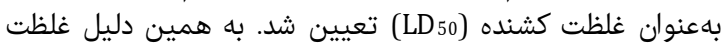

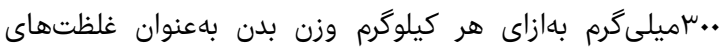

درمانى انتخاب شد[27].
با توجه به اينكه ميزان ترشح هورمونها و نوروهورمونها وابسته به

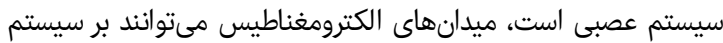

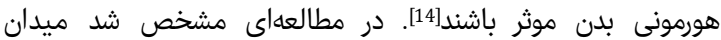

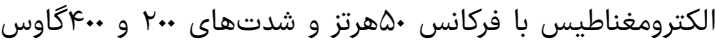

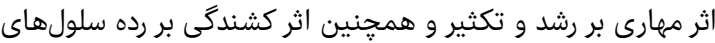

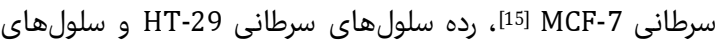

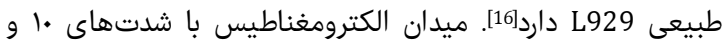

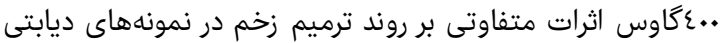

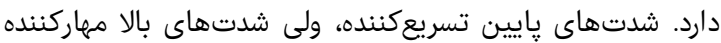

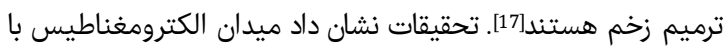

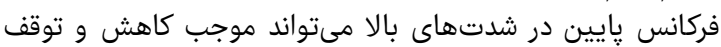

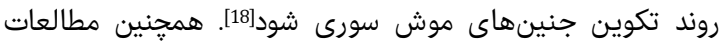

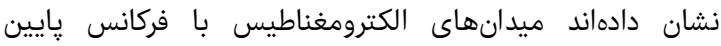

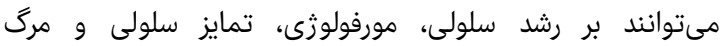

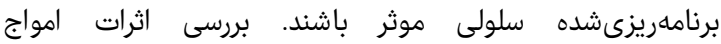

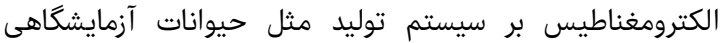

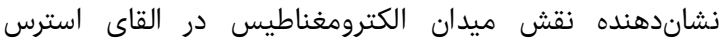

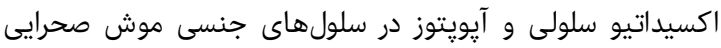

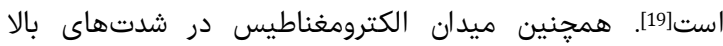

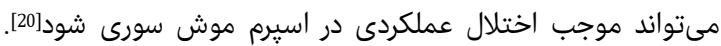

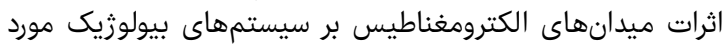

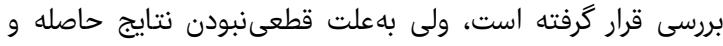

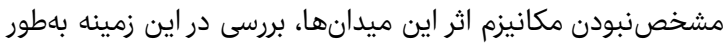
فعال ادامه دارد.

تورمريك (Turmeric) يا زردجوبه نام عاميانه كوركوما لونكا

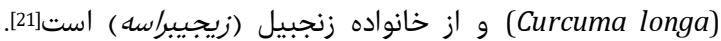

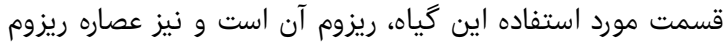

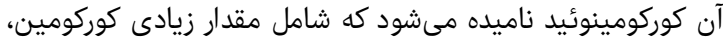

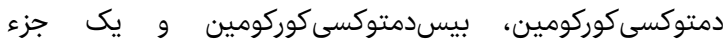

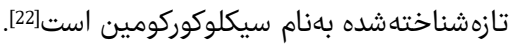

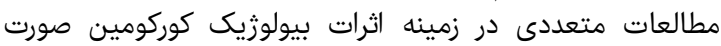

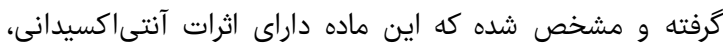

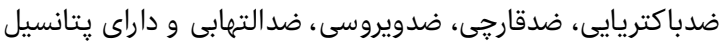

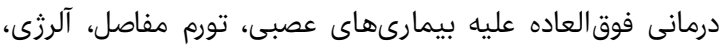

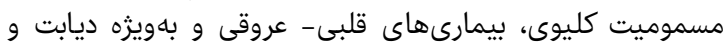

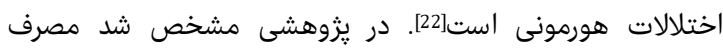

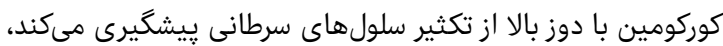

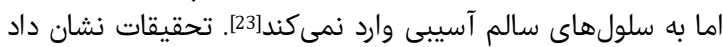

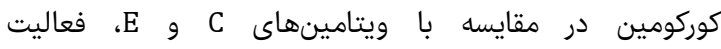
آنتى اكسيدانى بسيار قوىترى دارد و موجب مادي مهار سنتز راديكالهاي

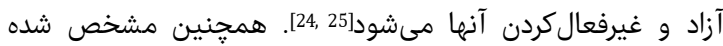

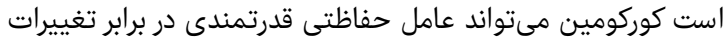

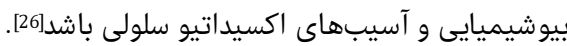

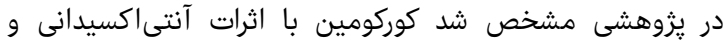

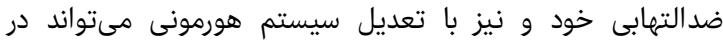

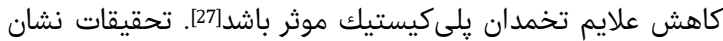

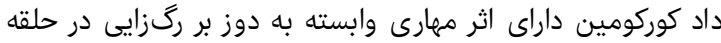

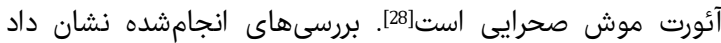

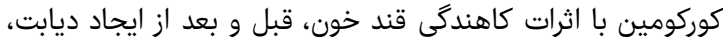

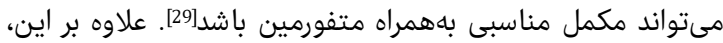

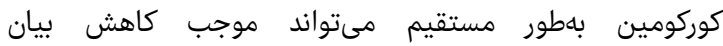

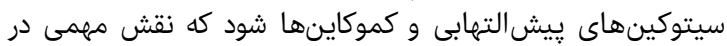


شكمى، مستقيماً از قلب موشها خونكيرى بهعمل آمد و به لوله

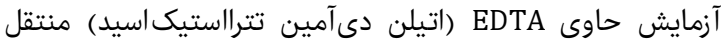

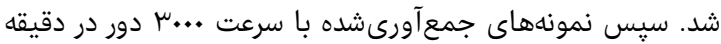

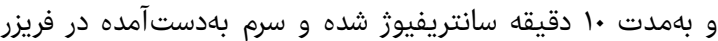

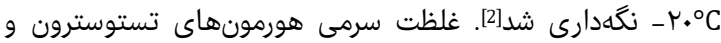

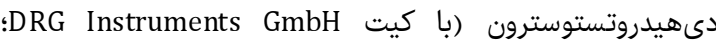

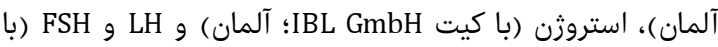

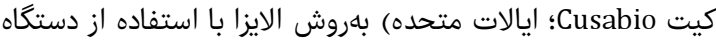

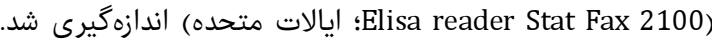
روش سنجش هورمونى براساس روش موجود در دفترجه راهنماى اندي

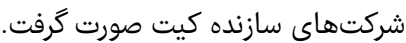

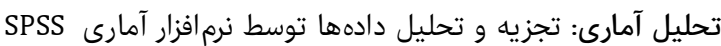

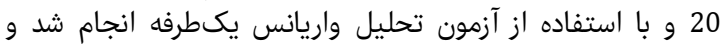

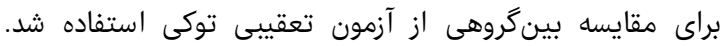

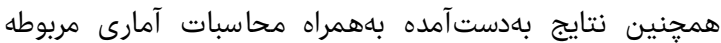
بلاصورت ميانكين آمارى بيان شد.

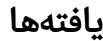
سطح سرمى هورمونهاى تستوسترون، دى ديدروتستوسترون،

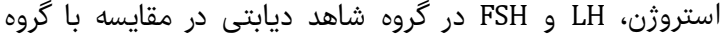

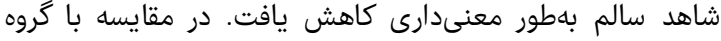

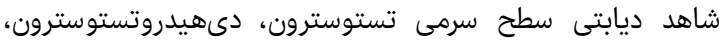

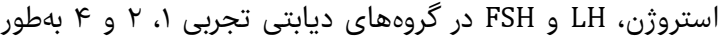

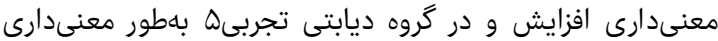

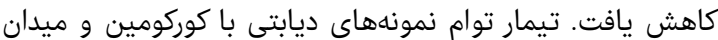

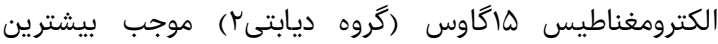

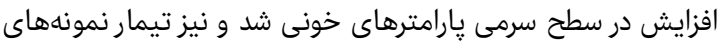

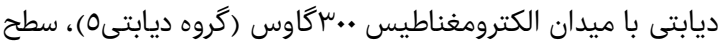

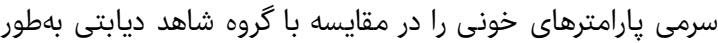

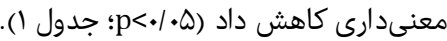

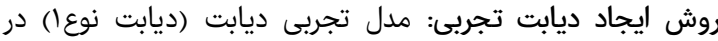

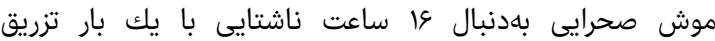

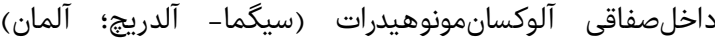

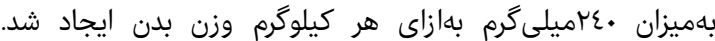

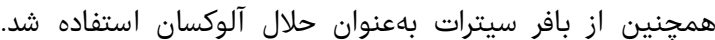

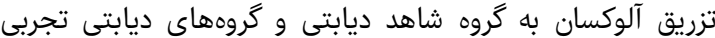

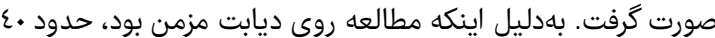

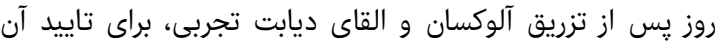

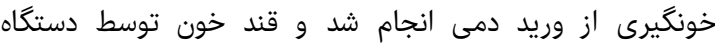

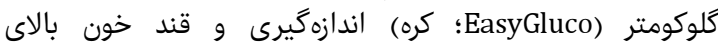

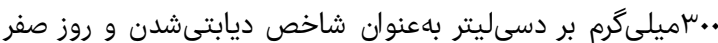

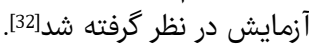

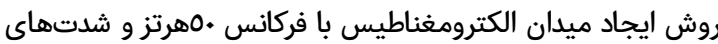

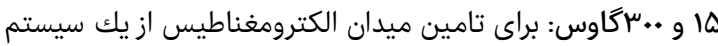

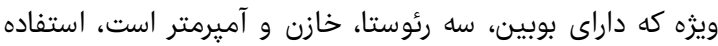

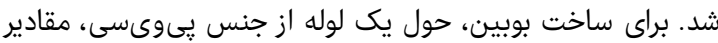

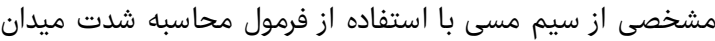

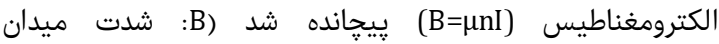

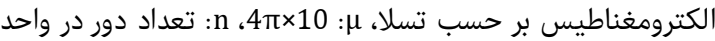

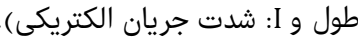

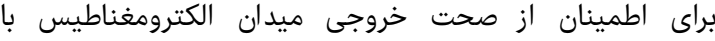

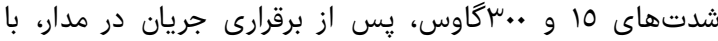

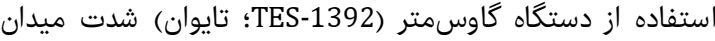

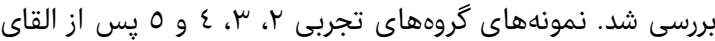

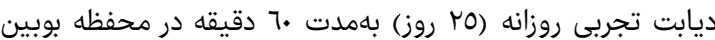

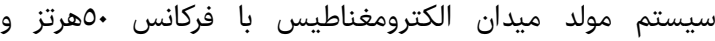

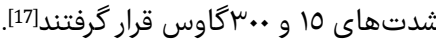

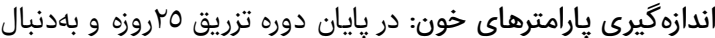

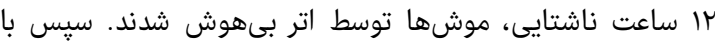
برش يوست در ناحيه شكم و قفسه سينه و از طريق بازئ بازكردن حفره

\begin{tabular}{|c|c|c|c|c|c|c|}
\hline كروه ديابتى0 & كروه ديابتىع & گروه ديابتى & كروه ديابتى & گروه ديابتى1 & كروه شاهد ديابتى & كروه شاهد سالم \\
\hline & & & & & \multicolumn{2}{|c|}{ سطح هورمون تستوسترون (نانومول در ليتر) } \\
\hline bef $\curlyvee / \wedge ৭ \pm \cdot / \wedge \vee$ & bd $\varepsilon / \Lambda \Lambda \pm l / \mu \wedge$ & $c \varepsilon / 1 \mu_{ \pm} / q \varepsilon$ & bc $\gamma / \varepsilon \mu_{ \pm} \pm / / \mu \gamma$ & 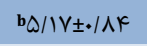 & $\mathrm{a} r / \Lambda r_{ \pm} \pm 1 / \cdot r$ & $q / V \cdot \pm r / \cdot \varepsilon$ \\
\hline befIV/AY $\pm 0 /$ YO & bd $ץ q / \mu O \pm \sum / 01$ & $c r o / 10 \pm \varepsilon / \mu^{\mu}$ & bc $\varepsilon_{0} / .0 \pm \Lambda / 01$ & $b \mu / / \varphi . \pm Q / q \mu$ & \multicolumn{2}{|c|}{ سطح هورمون دىهيدروتستوسترون (نانوكرم در دسىليتر) } \\
\hline & & & & & \multicolumn{2}{|c|}{ سطح هورمون استروثن (ييكوكرم در ميلىليتر) } \\
\hline bef $/ \mu / \Upsilon \Lambda \pm \varepsilon / V \Psi$ & bd $\varepsilon \cdot / 1 \| 0 / r \Lambda$ & $c \mu \mid / / r \pm r / / \Lambda$ & bc $\urcorner \Lambda / 1 \cdot \pm 1 \cdot / \mu_{0}$ & 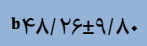 & 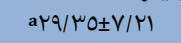 & $\Lambda \psi / \varepsilon 0 \pm \| / / \varepsilon \wedge$ \\
\hline & & & & & \multicolumn{2}{|c|}{ سطح هورمون LH (ميلىواحد در ميلىليتر) } \\
\hline 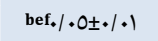 & 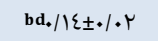 & $c . / 11 \pm . / \cdot r$ & bc. $/ Y \mid \pm \cdot / \cdot V$ & b. $/ \mid \varepsilon \pm \pm . / k$ & a. $/ .9 \pm . / .1$ & $\cdot / r \Lambda \pm \cdot / \cdot \vee$ \\
\hline & & & & & \multicolumn{2}{|c|}{ سطح هورمون FSH (ميلىواحد در ميلىليتر) } \\
\hline bef. $/ / 1 \pm \cdot / \cdot r$ & bd. $/ r \varepsilon \pm \cdot / \cdot \Lambda$ & $c . / 19 \pm . / \cdot \Lambda$ & $b_{\bullet} / \mu \mu_{ \pm \cdot / \cdot \Lambda}$ & b./ $/$ A.$\pm / .9$ & 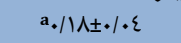 & $\cdot / \varepsilon \mu_{ \pm} \cdot / 11$ \\
\hline
\end{tabular}

فعاليت محور هييوفيز- گناد، هورمونهاى جنسى و ميزان بارورى موثر

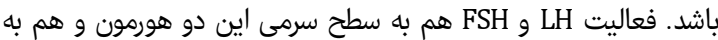

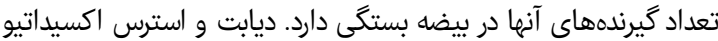

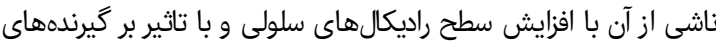

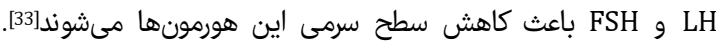

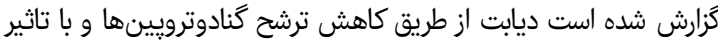

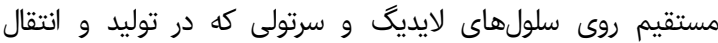

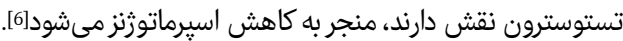

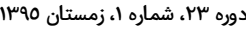

بحث

در يزوهش حاضر، اثر كوركومين و ميدان الكترومغناطيس با بسامد

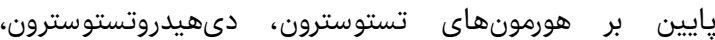

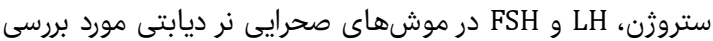

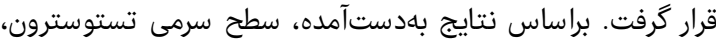

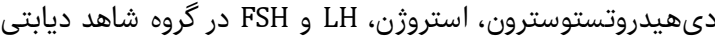

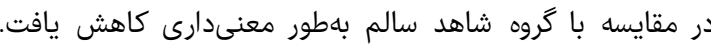
تحقيقات نشان داد ديابت منجر به ايجاد استرس اكسيداتيو سلولى دراني در

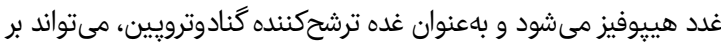
فصل نامه علمى - يُزوهشى افق دانش 


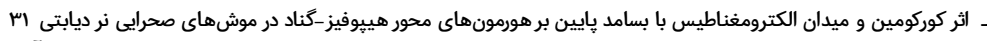

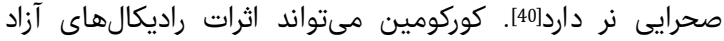

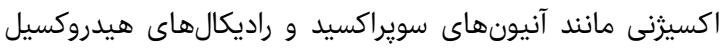

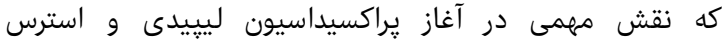

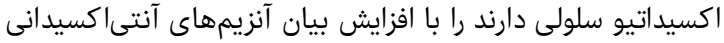

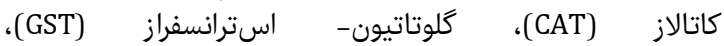

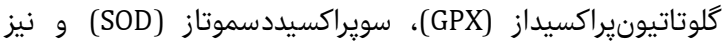

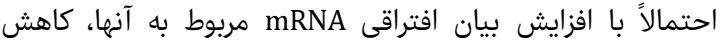

دهد[41].

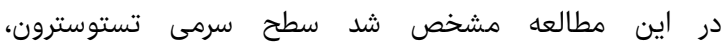

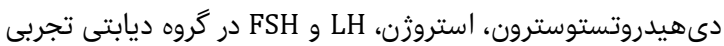

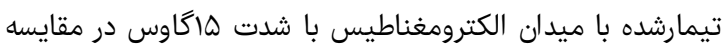

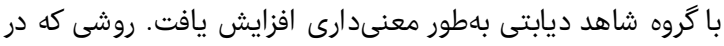

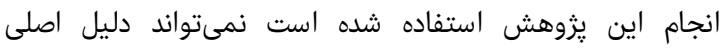

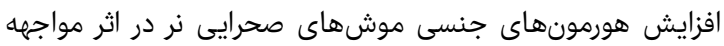

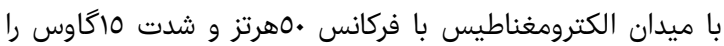

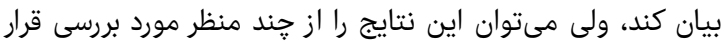

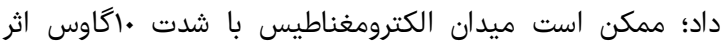

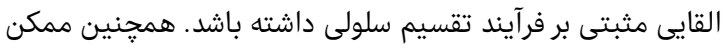

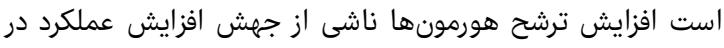

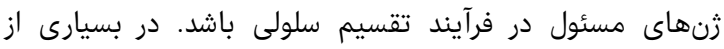

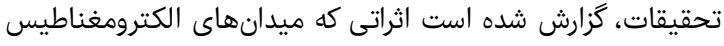

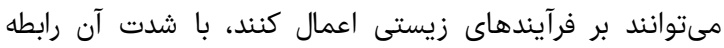

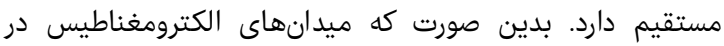

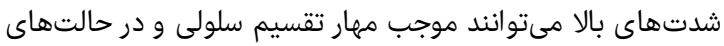

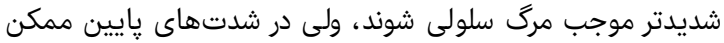

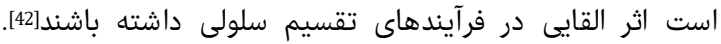

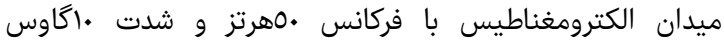

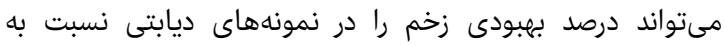

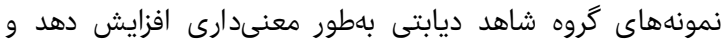

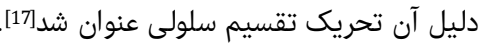

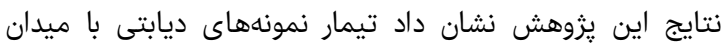

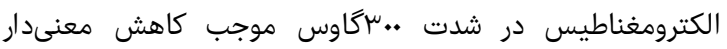

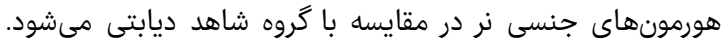

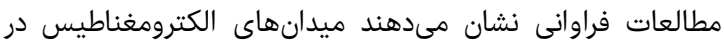

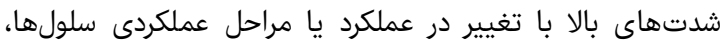

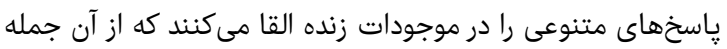

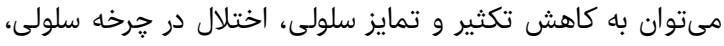

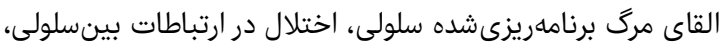

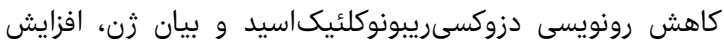

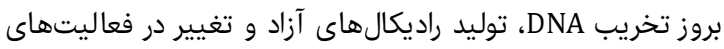

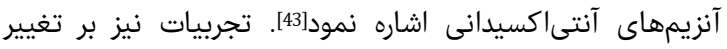

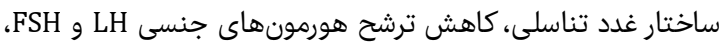

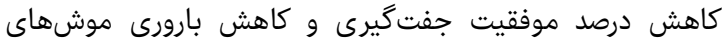

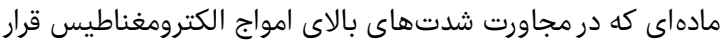

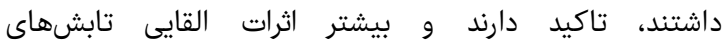

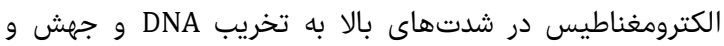

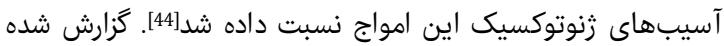

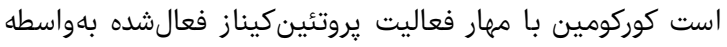

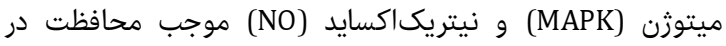

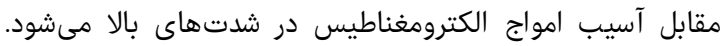

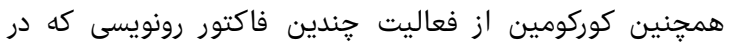
سرطانزايى دخالت دارند ممانعت مىكنين از فعلد از جمله اين فاكتورين روني
با توجه به نتايج بهدستآمده در يزوهش حاضر، سطح سرمى آرى

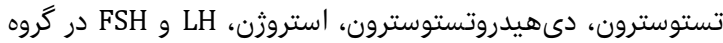

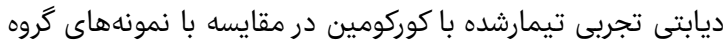

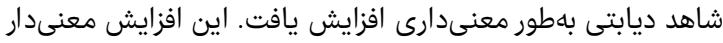

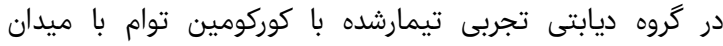

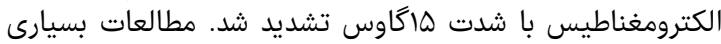

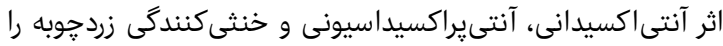

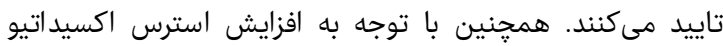

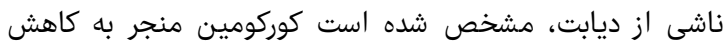

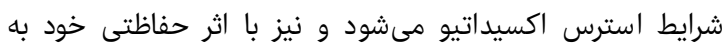

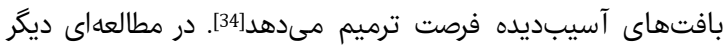

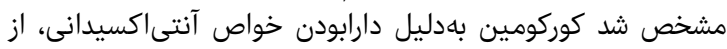

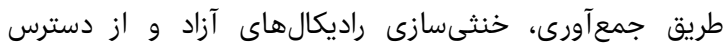

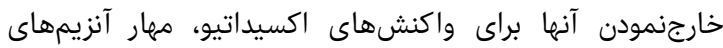

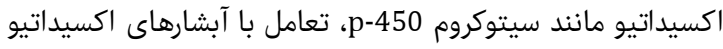

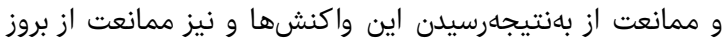

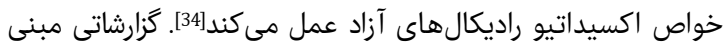

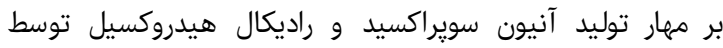

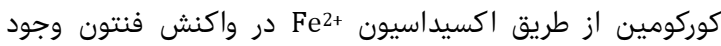

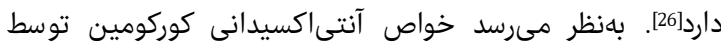

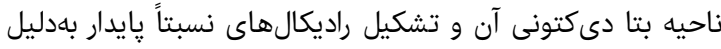

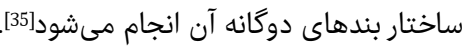

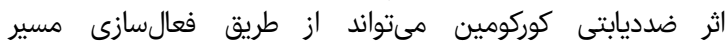
AMPK/ACC

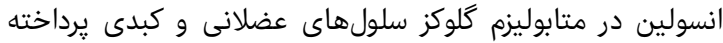

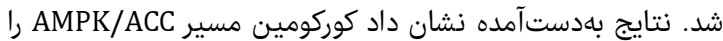

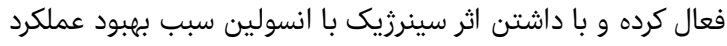

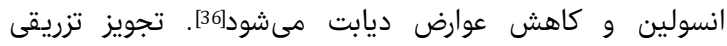

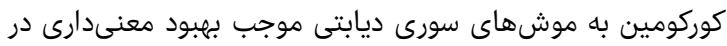

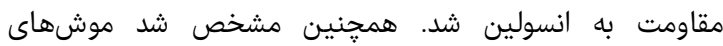

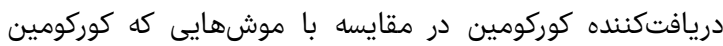

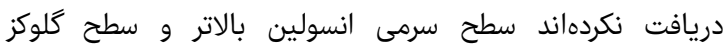

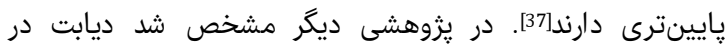

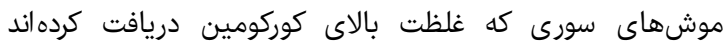

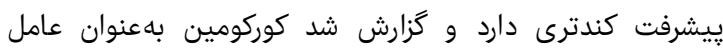

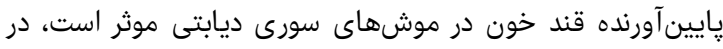

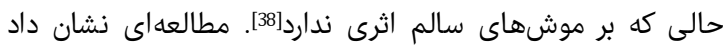

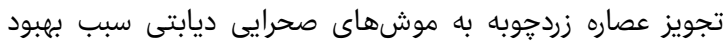

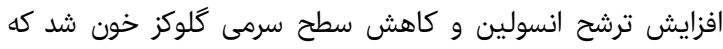

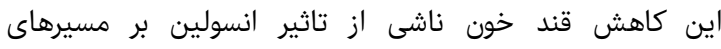

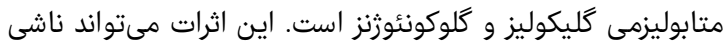

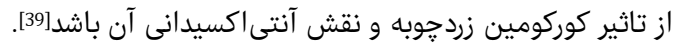

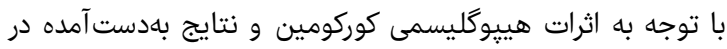

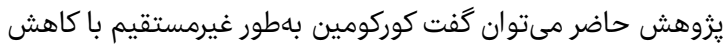

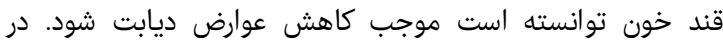

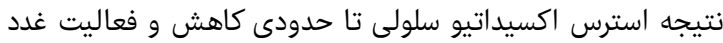

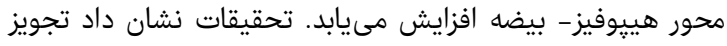

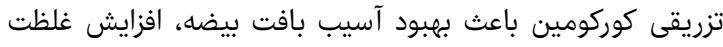

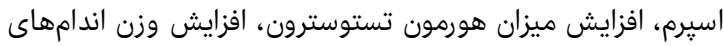

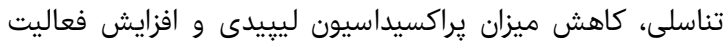

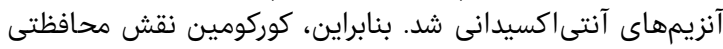

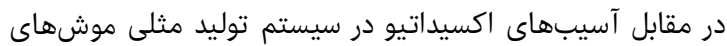


منابع مالى: هزينههاى اجراى طرح توسط باشكاه يزوهشكگران جوان و نخبكان دانشكاه آزاد اسلامى تامين و تخصيص يانيان يافته

1- Alzaman N, Ali A. Obesity and diabetes mellitus in the Arab world. J Taibah Univ Med Sci. 2016;11(4):301-9.

2- Rahbarian R, Sadooghi SD. Investigating the effects of aqueous extract of asafoetida resin on the serum level of insulin and blood glucose in type 1 diabetic rats. J Shahrekord Univ Med Sci. 2014;16(3):16-21. [Persian] 3- Najar A, Piryae A, Babaei S, Bayat M. Effect of pentoxifylline on Serotoli and Leidig cells count of experimentally induced type 1 diabetes in male rats. Ann Mil Health Sci Res. 2013;11(3):188-95. [Persian] 4- Jin J, Yang W. Molecular regulation of hypothalamuspituitary-gonads axis in males. Gene. 2014;551(1):15-25. 5- Holdcraft R.W, Braun R.E. Hormonal regulation of spermatogenesis. Int J Androl. 2004;27(6):335-42.

6- Shayakhmetova GM, Bondarenko LB, Matvienko AV, Kovalenko VM. Correlation between spermatogenesis disorders and rat testes CYP2E1 mRNA contents under experimental alcoholism or type I diabetes. Adv Med Sci. 2014;59(2):183-9.

7- Nah WH, Koh IK, Ahn HS, Kim MJ, Kang H, Jun JH, et al. Effect of Spirulina maxima on spermatogenesis and steroidogenesis in streptozotocin-induced type I diabetic male rats. Food Chem. 2012;134(1):173-9.

8- Rochette L, Zeller M, Cottin Y, Vergely C. Diabetes, oxidative stress and therapeutic strategies. Biochim Biophys Acta. 2014;1840(9):2709-29.

9- Spark RF. Testosterone, diabetes mellitus, and the metabolic syndrome. Curr Urol Rep. 2007;8(6):467-71.

10- Rho H, Lee J, Kim H, Park B, Park J. Protective mechanism of glucose against alloxan-induced B-cell damage: Pivotal role of ATP. Exp Mol Med. 2000;32(1):12-7.

11- Yamanouchi T, Moromizato H, Kojima SH, Shinohara T, Sekino N, Minoda S, et al. Prevention of diabetes by thymic hormone in alloxan-treated rats. Eur J Pharmacol. 1994;257(1-2):39-46.

12- Shahbazi-Gahrouei D, Shiri L, Alaei H, Naghdi N, Kermani S, Afrouzi H, et al. the effect of extremely lowfrequency magnetic fields on the level of serotonin metabolite in the raphe nuclei of adult male rat. J Isfahan Med Sch. 2014;32(298):1354-62.

13- Canedo L, Cantu RG, Hernandez R. Magnetic field exposure during gestation: pineal and cerebral cortex serotonin in the rat. Int J Dev Neurosci. 2003;21(5):263-6. 14- Koyu A, Cesur G, Ozguner F, Akdogan M, Mollaoglu H, Ozen S. Effects of $900 \mathrm{MHz}$ electromagnetic field on TSH and thyroid hormones in rats. Toxicol Lett. 2005;157(3):257-62.

15- Sadooghi SD, Zafar Balanezhad S, Nezhad Shahrokh Abadi $\mathrm{K}$, Baharara J. Investigating the effects of low frequency electromagnetic field on MCF-7 cancer cell line. Urmia Med J. 2014;25(5):444-52. [Persian]

16- Sadooghi SD. Investigating the effects of lowfrequency electromagnetic field on HT-29 cancer cell line. Feyz. 2013;17(5):413-21. [Persian]

17- Sadooghi SD. Investigating the effects of low frequency electromagnetic field on wound healing in diabetic rats. J Rafsanjan Univ Med Sci. 2014;13(3):207-22. 18- Rahbarian R, Sadooghi SD. Investigating the effect of electromagnetic field with low frequency on development of two-cell nmri mice embryo (in vitro). Urmia Med J. 2014;25(1):67-75. [Persian]
NF-KB و NF-KB

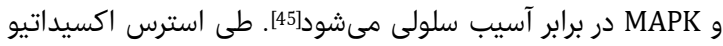

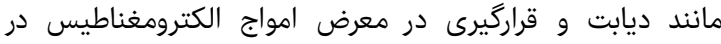

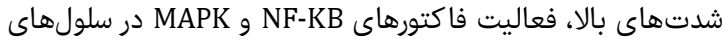
سرتولى افزايش مىيابد و بهدنبال آن استرس اكسيداتيو ايجادشده فيدا

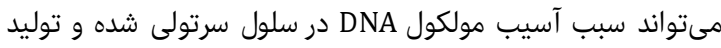

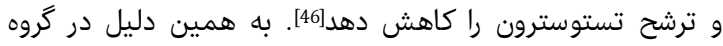

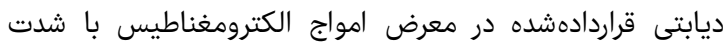

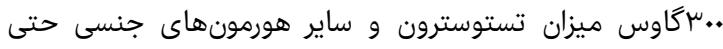

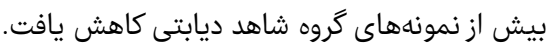

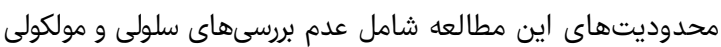

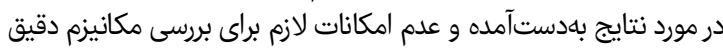

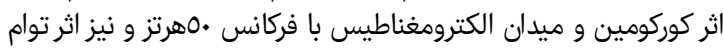

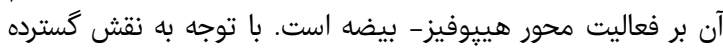

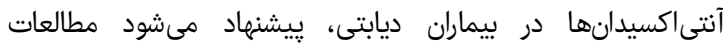

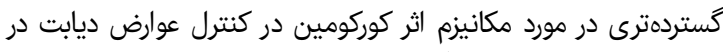

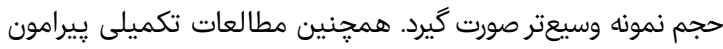

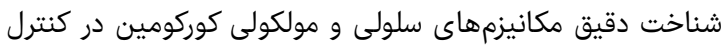

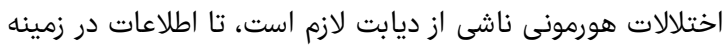

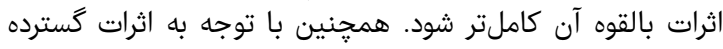

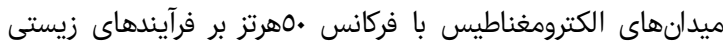

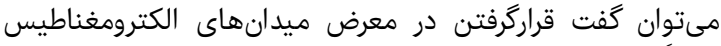

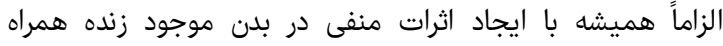

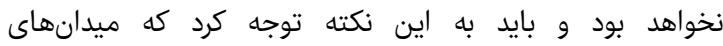

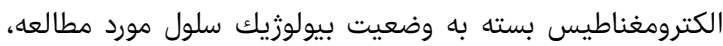

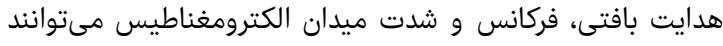

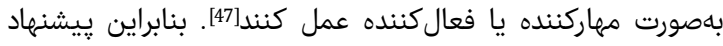

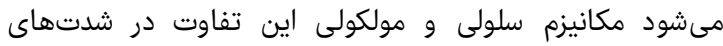

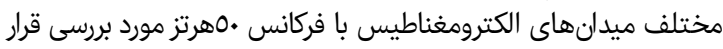

\section{نتيجه كيرى}

كوركومين با تاثير بر فعاليت محور هييوفيز- بيضه، ميزان ترشح

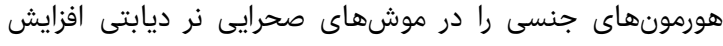

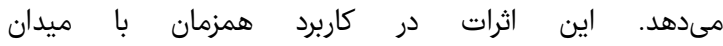

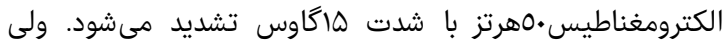

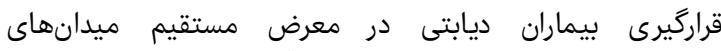

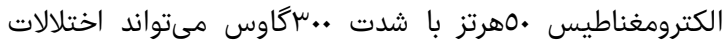

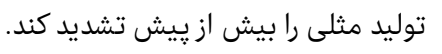

تشكر و قدردانى: بدين وسيله نويسنده مقاله از باشگًاه يزوهشگگران

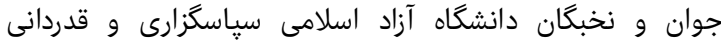

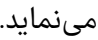
تاييديه اخلاقى: رعايت تمامى حقوق حيوانات آزمايشگاهى در

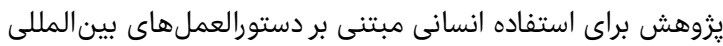

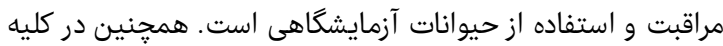

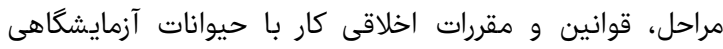

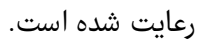
تعارض منافع: موردى از طرف نويسندگان بيان نشده است.

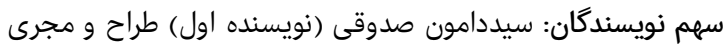

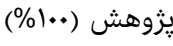


اثر كوركومين و ميدان الكترومغناطيس با بسامد پايين بر هورمونهاى محور هيِوفيز-كناد در موشهاى صحرايى نر ديابتى سبر

hypogonadism. Nutr Metab Cardiovasc Dis 2016;26(1):53-9.

34- Kumar P, Padi SS, Naidu PS, Kumar A. Possible neuroprotective mechanisms of curcumin in attenuating 3-nitropropionic acid-induced neurotoxicity. Methods Find Exp Clin Pharmacol. 2007;29(1):19-25.

35- Sumanont Y, Murakami Y, Tohda M, Vajragupta O, Matsumoto K, Watanabe H. Evaluation of the nitric oxide radical scavenging activity of manganese complexesof curcumin and its derivative. Biol Pharm Bull. 2004;27(2):170-3.

36- Gholami F, Mohiti Ardakani J, Moradi A, Danesh Pouya F. Anti-diabetic effect of curcuma longa extract via noninsulin dependent cellular pathway AMPK. J Shahid Sadoughi Univ Med Sci. 2013;21(3):311-8. [Persian]

37- Seo KI, Choi MS, Jung UJ, Kim HJ, Yeo J, Jeon SM, et al. Effect of curcumin supplementation on blood glucose, plasma insulin, and glucose homeostasis related enzyme activities in diabetic db/db mice. Mol Nutr Food Res. 2008;52(9):995-1004.

38- Chuengsamarn S, Rattanamongkolgul S, Luechapudiporn R, Phisalaphong C, Jirawatnotai S. Curcumin extract for prevention of type 2 diabetes. Diabetes Care. 2012;35(11):2121-7.

39- Ayoubi AR, Valizadeh R, Omidi A, Abolfazli M. Evaluation of Turmeric (Curcuma longa) effects in preventing consequences of lead acetate in male rats. J Birjand Univ Med Sci. 2014;21(1):68-76. [Persian]

40- Chandra AK, Chatterjee A, Ghosh R, Sarkar M. Effect of curcumin on chromium-induced oxidative damage in male reproductive system. Environ Toxicol Pharmacol. 2007;24(2):160-6.

41- Noorafshan A, Karbalay-Doust S, Valizadeh A, Aliabadi E, Mirkhani H. Ameliorative effects of curcumin on the seminiferous epithelium of curcumin on the seminiferous epithelium in metronidazole-treated mice: a stereological study. Toxicol Pathol. 2010;38(3):366-71. 42- Baharara J, Zahedifar Z. The effect of low-frequency electromagnetic fields on some biological activities of animals. Arak Med Univ J. 2012;15(7):80-93. [Persian] 43- Focke F, Schuermann D, Kuster N, Schär P. DNA fragmentation in human fibroblasts under extremely low frequency electromagnetic field exposure. Mutat Res. 2010;683(1-2):74-83.

44- Baharara J, Parivar K, Oryan Sh, Ashraf AR. Effects of low frequency electromagnetic fields on gonads and fertility of female Balb/c mouse. Arak Med Univ J. 2006;9(2):1-11. [Persian]

45- Biswas SK, Mcclure D, Jimenez LA, Megson IL, Rahman I. Curcumin induces glutathione biosynthesis and inhibit NF-kappaB activation and interleukin-8 release in alveolar epithelial cells: Mechanism of free radical scavenging activity. Antioxid Redox Signa. 2005;7(1-2):32-41.

46- Gilmore TD. Introduction to NF-kappaB: Players, pathways, perspectives. Oncogene. 2006;25(51):6680-4.

47- Monsefi M, Baha-al-dini A, Pirooz M, Haghighi S.

Effect of electromagnetic field on ovary and sex hormones of female rat. J Birjand Univ Med Sci. 2004;11(4):9-15. [Persian]
19- Rajaei F, Mohammadian A. Effects of extremely low frequency electromagnetic field on mouse liver histology. J Qom Univ Med Sci. 2013;6(4):8-13. [Persian] 20- Saadat I, Sedaghat Z, Afhami M, Bahaoddini A, Saadat M. Effect of a $50-\mathrm{Hz}$ electromagnetic field on the gene expression of glutathione s-transferase T1 (Gstt1) in the testis and liver of male rats. Comp Clin Pathol. 2010;19(2):211-14.

21- Kamali E, Ghaedi K, Karimi P, Kheradmand P, Tavassoli M. Biological and Anticancer Effects of Curcumin. J Isfahan Med Sch. 2014;31(265):2097-112. [Persian]

22- Maheshwari RK, Singh AK, Gaddipati J, Srimal RC. Multiple biological activities of curcumin: A short review. Life Sci. 2006; 78(18):2081-7.

23- Anand P, Sundaram C, Jhurani S, Kunnumakkara AB, Aggarwal BB. Curcumin and cancer: An "old-age" disease with an "age-old" solution. Cancer Lett. 2008;267(1):133-64.

24- Kant V, Gopal A, Pathak NN, Kumar P, Tandan SK, Kumar D. Antioxidant and anti-inflammatory potential of curcumin accelerated the cutaneous wound healing in streptozotocin-induced diabetic rats. Int Immunopharmacol. 2014;20(2):322-30.

25- Toda S, Miyase T, Arichi H, Tanizawa H, Takino Y. Natural antioxidants. III. Antioxidative components isolated from rhizome of Curcuma longa L. Chem Pharm Bull. 1985;33(4):1725-8.

26- Zargari M, Ahmadi S, Shabani S, Mahrooz A. Protective effect of curcumin on the superoxide dismutase and catalase activity in kidney of acetaminophen-exposed rats. J Mazandaran Univ Med Sci. 2013;22(97):74-83. [Persian]

27- Nabiuni M, Mohammadi S, Kayedpoor P, Karimzadeh L. The effect of curcumin on the estradiol valerateinduced polycystic ovary in rats. Feyz. 2015;18(6):515-23. [Persian]

28- Baharara J, Mousavi M, Ramezani T. Effect of curcumin on angiogenesis in aortic ring model of the wistar rat. J Shahid Sadoughi Univ Med Sci. 2014;22(3):1226-36. [Persian]

29- Ameli H, Moini-Zangani T, Masoudnia F, Sabetkasaei $\mathrm{M}$. The comparison of curcumin's effect with or without metformin on blood glucose levels in diabetic rats. Pejouhandeh. 2015;19(6):312-19. [Persian]

30- Jagetia GC, Aggarwal BB. "Spicing up" of the immune system by curcumin. J Clin Immunol. 2007;27(1):19-35.

31- Roghani Dehkordi F, Roghani M, Baluchnejad Mojarad T. The effect of curcumin on serum level of aspartate and alanine amoinotransferase and cardiac level of oxidative stress markers in diabetic rats. Pejouhandeh. 2012;17(1):18-25. [Persian]

32- Rahbarian R, Sepehri Moghadam H, Sadoughi SD. Effect of aqueous extract of launaea acanthodes on open skin wound in diabetic rats. Horizon Med Sci. 2016;21(4):221-2. [Persian]

33- Tirabassi G, Chelli FM, Ciommi M, Lenzi A, Balercia G. Influence of the hypothalamic-pituitary-adrenal axis dysregulation on the metabolic profile of patients affected by diabetes mellitus-associated late onset 